\title{
Entwicklung eines voltammetrischen In-situ- Sensorsystems zum Nachweis von As(III) und As(V)
}

\author{
Janek Weißpflog ${ }^{1}$, Kathrin Trommer ${ }^{1}$, Johannes Schwarz ${ }^{1}$ und Michael Mertig ${ }^{1,2}$ \\ ${ }^{1}$ Kurt-Schwabe-Institut für Mess- und Sensortechnik Meinsberg e.V., Waldheim, Deutschland \\ ${ }^{2}$ Professur für Physikalische Chemie, Mess- und Sensortechnik, Technische Universität Dresden, Dresden, \\ Deutschland \\ Kontakt: janek.weisspflog@ksi-meinsberg.de
}

\section{Einleitung}

In der bis 1990 betriebenen Bergbaugrube in Ehrenfriedersdorf wurde hauptsächlich Zinnerz abgebaut; zwei Spülhalden entstanden als Resultat dieser Zinnerzförderung. Nach der Schließung und Verwahrung der Grube wurden die bergmännischen Hohlräume geflutet. Die Grubenwässer weisen laut Hösel et al. [1] in der Folge eine hohe As- und FeBelastung auf. In den Spülhalden wurden die Rückstände der Zinnaufbereitung der letzten Bergbauperiode seit 1942 aufgehaldet. Die Haupterzminerale der Lagerstätte Ehrenfriedersdorf sind Zinnstein (Kassiterit, $\mathrm{SnO}_{2}$ ), Arsenopyrit (FeAsS) und Löllingit $\left(\mathrm{FeAs}_{2}\right)$ [1]. Bevor eine Behandlungstechnologie zur Entfernung von Arsen aus Trinkwasser und Böden ausgewählt wird, muss in Erfahrung gebracht werden, welche Arsenspezies im Wasser oder Boden vorhanden ist. Die Mobilität und Toxizität von Arsenionen wird stark durch seine Speziation (Ladungszahl) beeinflusst. As(V) kommt in wässriger Lösung je nach pH-Wert in vier Formen vor: $\mathrm{H}_{3} \mathrm{AsO}_{4}$ (Arsenat, $\mathrm{pK}_{1}=2,2$; anionisch bei neutralem $\mathrm{pH}$ ), $\mathrm{H}_{2} \mathrm{AsO}_{4}{ }^{-}, \mathrm{HAsO}_{4}{ }^{2-}$ und $\mathrm{AsO}_{4}{ }^{3-}$. In ähnlicher Weise kommt $\mathrm{As}(\mathrm{III})$ in fünf Formen vor: $\mathrm{H}_{4} \mathrm{AsO}_{3}{ }^{+}, \mathrm{H}_{3} \mathrm{AsO}_{3}$ (Arsenit, $\mathrm{pK}_{1}=9,2$; undissoziiert bei neutralem $\mathrm{pH}$ ), $\mathrm{H}_{2} \mathrm{AsO}_{3}{ }^{-}, \mathrm{HAsO}_{3}{ }^{2-}$, und $\mathrm{AsO}_{3}{ }^{-}$. Die ionischen Formen von $\mathrm{As}(\mathrm{V})$ dominieren bei $\mathrm{pH}>3$, und $\mathrm{As}(\mathrm{III})$ ist bei $\mathrm{pH}<9$ neutral und bei $\mathrm{pH}>9$ ionisch [2]. Da die Nettoladung von As(III) bei natürlichen $\mathrm{pH}$-Werten von 6 $\leq \mathrm{pH} \leq 9$ neutral ist, lässt sich diese Form nicht leicht entfernen. As(III) gilt zudem als mobiler und giftiger für lebende Organismen. Dagegen zählen organische Arsenarten als sehr viel weniger gesundheitsschädlich und können vom Körper leicht ausgeschieden werden. Außerdem ist As(III) in Böden mobiler als $\mathrm{As}(\mathrm{V})$. Organisches As ist im Vergleich zu anorganischen Formen von As weniger mobil. Daher ist die Umwandlung von $\mathrm{As}(\mathrm{III}) \rightarrow \mathrm{As}(\mathrm{V})$ ein kritischer Schritt jedes Arsenbehandlungsverfahrens.

Die durchschnittliche Arsenkonzentration der Wässer in der Zinnerzgrube Ehrenfriedersdorf und deren Umgebung beträgt etwa 0,5 mg/l (vgl. TrinkwV: oberer Grenzwert $\left.c_{\max }(A s)=10 \mu \mathrm{g} / \mathrm{l}\right)$. Die Redoxstabilität von $\mathrm{As}(\mathrm{III})$ und $\mathrm{As}(\mathrm{V})$ hängt vom $\mathrm{pH}-$ Wert und EhWert in Lösung ab. Bei niedrigem $\mathrm{pH}$-Wert herrscht As(III) zwischen 0 und $500 \mathrm{mV}$ vor, wohingegen
As(V) oberhalb von $500 \mathrm{mV}$ stabil ist. Mit steigendem $\mathrm{pH}-$ Wert ist As(III) nur dann stabil, wenn der Eh-Wert kontinuierlich sinkt. Die Stabilität von As(V) nimmt mit steigendem $\mathrm{pH}$-Wert über $0 \mathrm{mV}$ zu [3-5]. Eisen und seine eventuelle Oxidation sind wichtige Einflussfaktoren für die Arsenspeziation in wässrigen Umweltproben. Ein niedriger Eisengehalt, wie es in vielen Grundwässern und Grubenentwässerungen mit hohem $\mathrm{pH}-$ Wert der Fall sein könnte, kann bei einem pH-Werten von über 4 eine beträchtliche Menge Arsenat ausgefällt werden [6].

Sowohl As(V) als auch As(III) bilden mit vielen Metallionen Chelate und Ausfällungen. $\mathrm{Ca}_{3}\left(\mathrm{AsO}_{4}\right)_{2}$ ist die stabilste As(V)-Spezies in gut oxidierten alkalischen Umgebungen. Unter reduzierenden Bedingungen und hohen Mn-Konzentrationen in den Böden wird die Löslichkeit von As durch $\mathrm{Mn}_{3}\left(\mathrm{AsO}_{4}\right)_{2}$ kontrolliert [3]. Samadzadeh et al. beschrieb den Sättigungsindex als geeignetes Kriterium für den Vergleich der Tendenz verschiedener Metalle, mit Arsen auszufallen [7]. Demnach bildet As(V) insbesondere im $\mathrm{pH}$-Bereich von $4-12$ feste Verbindungen mit vielen metallischen Kationen. Weiterhin wurde ein Fixierungsprozess beschrieben, bei dem mit $\mathrm{As}(\mathrm{V})$ kontaminierte Feststoffe durch die 1:1Zugabe von Eisensulfat $\left(\mathrm{FeSO}_{4} \cdot 4 \mathrm{H}_{2} \mathrm{O}\right)$-Lösung behandelt werden, um Eisen(III)-arsenat zu erzeugen. Es ist offensichtlich, dass die Verfügbarkeit von $\mathrm{O}_{2}$ einer der Schlüsselfaktoren ist, die den Prozess steuern. $\mathrm{FeAsO}_{4}$ ist eine unlösliche Verbindung [3]:

$$
\begin{aligned}
& \mathrm{Fe}\left(\mathrm{H}_{2} \mathrm{O}_{6}\right)^{2+} \rightarrow \mathrm{Fe}\left(\mathrm{H}_{2} \mathrm{O}\right)_{6}{ }^{3+}+\mathrm{e}^{-} \\
& \mathrm{Fe}^{3+}+\mathrm{AsO}_{4}{ }^{3-} \rightarrow \mathrm{FeAsO}_{4}
\end{aligned}
$$

Arsen kann also entweder direkt durch Metallkationen oder durch Co-Präzipitation mit metallischen Hydroxiden aus der Lösung ausgefällt werden. Bei der konventionellen Koagulation bilden sich große, nicht dispergierte Partikel aus einem Kolloid, z. B. hydratisiertes $\mathrm{Fe}_{2}\left(\mathrm{SO}_{4}\right)_{3}$ mit einem gelösten Stoff, z. B. $\mathrm{H}_{2} \mathrm{AsO}_{4}^{-}$[3].

Oberhalb des $\mathrm{pH}-$ Werts von $\mathrm{pH}=2,2$ ist die Ladung der Arsensäure (As(V)) negativ. In Böden und Sedimenten sind die Oberflächen von Quarzkörnern und sekundären Tonmineralien mit pH-abhängigen Metalloxiden überzogen, deren Ladung positiv ist, bis etwa $\mathrm{pH}$ 8, wo der Nullpunkt der Ladung der meisten Aluminium- (Al) und Eisenoxide (Fe(III)) 
liegt. Arsenat wird daher elektrostatisch von Oberflächen aus Ferrihydrit, Goethit, Hämatit oder $\mathrm{Al}_{2} \mathrm{O}_{3}$ angezogen. Arsenat ist daher in der Umwelt weniger mobil als das nichtionische As(III). Die Adsorption von Arsenat nimmt mit abnehmendem pH-Wert zu, während das Adsorptionsmaximum von As(III) im Allgemeinen in der Nähe seiner ersten Dissoziationskonstante $(9,22)$ liegt [3]. Die meisten derzeit angewandten Verfahren zur Entfernung von Arsen aus Trinkwasser beruhen auf der Oxidation, gefolgt von der Adsorption oder Co-Präzipitation von $\mathrm{As}(\mathrm{V})$ mit Eisen- und anderen Metalloxiden. Eine elektrochemische Untersuchung des Verhaltens von Arsen in wässrigen Lösungen wird das Verständnis für diese Reaktionen verbessern. Dies ist ein wichtiger Schritt zur Optimierung des Prozesses und zur effizienteren Entfernung von Arsen.

Für die Sensorik umweltrelevanter Schwermetallionen im Wasser sind elektrochemische Bestimmungsmethoden wie Voltammetrie und Potentiometrie gut etabliert. Beispielsweise wurden Elektroden für die spezifische Detektion von $\mathrm{Cu}^{2+}, \mathrm{NO}_{3}{ }^{-}$und $\mathrm{NH}_{4}{ }^{+}$entwickelt und extensiv charakterisiert $[8,9]$. Für eine effektive Anwendung der mittels Siebdrucktechnik hergestellten Elektroden sowie von miniaturisierten Stabelektroden wurden zudem miniaturisierte Referenzelektroden entwickelt.

In diesem Beitrag werden neuartige Siebdruckelektroden mit einer Drei-Elektroden-Anordnung beschrieben. Als Detektionsmethoden werden die Messtechniken Cyclovoltammetrie (CV) SquareWave-Anodic-Stripping-Voltammetrie (SWASV), Differential-Pulse-Anodic-Stripping-Voltammetrie

(DPASV) sowie Kathodic-Stripping-Voltammetrie (CSV) unter Verwendung eines tragbaren elektrochemischen Analysegeräts für die Untersuchungen eingesetzt.

\section{Methoden und Materialien}

Alle verwendeten Reagenzien waren von analytischer Qualität und wurden ohne weitere Aufbereitung verwendet. Alle wässrigen Lösungen wurden mit ultrareinem Wasser (Leitfähigkeit kleiner $0,058 \mu \mathrm{S} / \mathrm{cm}$ bei $298 \mathrm{~K}$ ) aus einem ThermoScientific MicroPure UV-Wasserreinigungssystem hergestellt. Standards von As(III) und As(V) wurden von SigmaAldrich bezogen, ebenso wie $\mathrm{Cu}(\mathrm{II})$ und $\mathrm{L}$ Ascorbinsäure. Die Chemikalien für die Herstellung der Acetat- und PBS-Pufferlösungen wie Eisessig, Salzsäure, Natriumhydroxid und diNatriumhydrogenphosphat wurden bei Carl Roth erworben. Kaliumdihydrogenphosphat stammt von der Firma Laborchemie Apolda.

\section{Elektrodenmaterial}

Bei den voltammetrischen Messungen wurde die Dreielektrodentechnik verwendet, mit $\mathrm{Pt}$ oder $\mathrm{Au}$ - basierter Arbeitselektrode, Gegenelektrode $(\mathrm{Pt})$ und $\mathrm{Ag} / \mathrm{AgCl}$ als Referenzelektrode (Abb. 1). In der Anordnung mit drei Elektroden auf einer gemeinsamen Substratoberfläche hat die Referenzelektrode die Aufgabe, mit inrem konstanten Potential für eine genauere Messung und Regelung zu sorgen. Das Potential der Arbeitselektrode wird gegen das der Referenzelektrode, durch die kein Strom fließt, gemessen. Damit wird der Fehler durch den ohmschen Spannungsabfall in der Lösung weitgehend eliminiert [10]. Um die Spannung wieder an das gewünschte Sollpotential anzugleichen, wird ein Strom von der Gegenelektrode zur Arbeitselektrode geschickt. Der eingesetzte Potentiostat PalmSens ${ }^{4}$ (Niederlande) wird mit der Software PSTrace (Version 5.8) gesteuert.

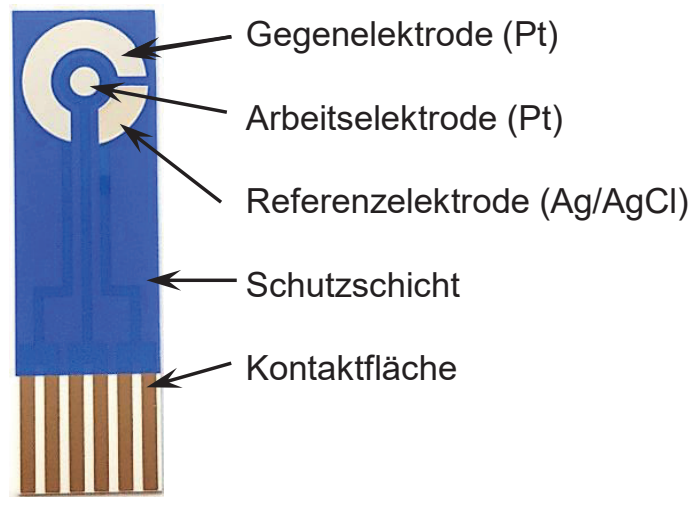

Abb. 1: Neu entwickelte Pt-basierte Siebdruckelektrode mit Drei-Elektroden-Anordnung (Pt-Arbeitselektrode, PtGegenelektrode und Ag/AgCl-Referenzelektrode).

\section{Voltammetrische Messungen}

Die Optimierung der elektrochemischen Parameter und die quantitative Bestimmung von $\mathrm{As}(\mathrm{III}), \mathrm{As}(\mathrm{V})$ und $\mathrm{Cu}(\mathrm{II})$ wurden mit Hilfe der SWASV durchgeführt. Vor jeder Messreihe wurde eine cyclovoltammetrische Messung mit folgenden Parametern $\left(E_{\text {start }}\right.$ $=1,5 \mathrm{~V} ; \mathrm{E}_{\text {vertex } 1}=-1,5 \mathrm{~V} ; \mathrm{E}_{\text {vertex } 2}=1,5 \mathrm{~V} ; \mathrm{E}_{\text {step }}=0,1$ V) durchgeführt. Anschließend wurden SWASVMessungen mit den angegebenen Messbedingungen durchgeführt bis die Basislinie reproduzierbar war. Die Bestimmung von $\mathrm{As}(\mathrm{III}), \mathrm{As}(\mathrm{V}), \mathrm{Cu}(\mathrm{II})$, $\mathrm{Fe}(\mathrm{III})$ und $\mathrm{Zn}$ (II) erfolgte nach elektrochemischer Reinigung und Aktivierung der Elektrodenoberfläche durch Anlegen eines Anreicherungspotentials $\left(E_{\text {dep }}\right)$ von $-1,5 \mathrm{~V}$ bei einer Anreicherungszeit ( $\mathrm{t}_{\mathrm{dep}}$ ) von 120 s. Die Anreicherung erfolgte unter Rühren, wobei ein Magnetrührer (Automatic Stirrer including Switch Box, EKTechnologies $\mathrm{GmbH}$, Wesel) zum Einsatz kam. Nach einer Standzeit von $10 \mathrm{~s}$ wurden Voltammogramme aufgenommen, während der Potentialbereich in der voltammetrischen Zelle gegen eine $\mathrm{Ag} / \mathrm{AgCl}$-Elektrode von $-1,5 \mathrm{~V}$ bis $+1,5 \mathrm{~V}$ gescannt wurde. Die voltammetrischen Parameter für 
die SWV waren Amplitude $=20 \mathrm{mV}$ und Frequenz $=$ $25 \mathrm{~Hz}$. Für jede Messung wurden Doppel-Scans aufgezeichnet. Die Experimente wurden bei Raumtemperatur durchgeführt. Die dargestellten Voltammogramme wurden nicht basislinienkorrigiert.

\section{Ergebnisse}

\section{CV-Untersuchungen von As in unterschiedlichen Elektrolytlösungen und bei $\mathrm{pH}$-Werten}

Voltammetrische Messungen werden durch die von den jeweiligen Elektrolyten abhängigen Potentialgrenzen bestimmt. Mit zunehmend niedrigerem $\mathrm{pH}$ Wert wird die Bildung von $\mathrm{H}_{2}$ bei negativem Anreicherungspotential gefördert. Je niedriger der $\mathrm{pH}$ Wert, desto mehr verlagert sich die $\mathrm{H}_{2}$-Bildung in positivere Bereiche. Aus diesem Grund wird die Verwendung von Anreicherungspotentialen, die negativer als $-2,0 \mathrm{~V}$ bei $\mathrm{pH} 12$ und negativer als $-0,5 \mathrm{~V}$ bei $\mathrm{pH} \sim 2$ sind, gegen $\mathrm{Ag} / \mathrm{AgCl}$ nicht empfohlen. Dagegen fördert ein zu hohes Potential, z. B. bei einer Pre-Konditionierung bzw. Reinigung, die Oxidation der Elektrodenoberflächen.

Abb. 2 zeigt Voltammogramme in Lösungen mit $\mathrm{As}(\mathrm{III})=1 \mathrm{mg} / \mathrm{l}$ in unterschiedlichen Elektrolyten und $\mathrm{pH}-$ Werten (PBS, $\mathrm{pH}=5 ; 6 ; 7$ und 8; Acetatpuffer, $\mathrm{pH}=4,6 ; \mathrm{HCl}, \mathrm{pH}=2,0)$. Im Potentialbereich $(-1,2 \rightarrow+1,5 \mathrm{~V})$ und bei den $\mathrm{pH}$-Werten $(\mathrm{pH}=2,0$ und $\mathrm{pH}=4,60)$ sind die stabilen Formen des Arsens elementares As, As(III) als $\mathrm{HAsO}_{2}, \mathrm{As}(\mathrm{V})$ als $\mathrm{H}_{3} \mathrm{AsO}_{4}$ $(\mathrm{pH}<3,60)$ oder $\mathrm{H}_{2} \mathrm{AsO}_{4}{ }^{-}(3,60<\mathrm{pH}<7,26)$ und Arsingas $\left(\mathrm{AsH}_{3}\right)$. Mögliche Reaktionen, sind nachfolgend zusammengefasst [11]:

$$
\begin{aligned}
& \mathrm{H}_{3} \mathrm{AsO}_{4}+2 \mathrm{H}^{+}+2 \mathrm{e}^{-} \rightarrow \mathrm{HAsO}_{2}+2 \mathrm{H}_{2} \mathrm{O} \\
& \mathrm{H}_{2} \mathrm{AsO}_{4}^{-}+3 \mathrm{H}^{+}+2 \mathrm{e}^{-} \rightarrow \mathrm{HAsO}_{2}+2 \mathrm{H}_{2} \mathrm{O} \\
& \mathrm{HAsO}_{2}+3 \mathrm{H}^{+}+3 \mathrm{e}^{-} \rightarrow \mathrm{As}+2 \mathrm{H}_{2} \mathrm{O} \\
& \mathrm{H}_{3} \mathrm{AsO}_{4}+5 \mathrm{H}^{+}+5 \mathrm{e}^{-} \rightarrow \mathrm{As}+4 \mathrm{H}_{2} \mathrm{O} \\
& \mathrm{As}+3 \mathrm{H}^{+}+3 \mathrm{e}^{-} \rightarrow \mathrm{AsH}_{3} \\
& \mathrm{HAsO}_{2}+6 \mathrm{H}^{+}+6 \mathrm{e}^{-} \rightarrow \mathrm{AsH}_{3}+2 \mathrm{H}_{2} \mathrm{O} \\
& \mathrm{H}_{3} \mathrm{AsO}_{4}+8 \mathrm{H}^{+}+8 \mathrm{e}^{-} \rightarrow \mathrm{AsH}_{3}+4 \mathrm{H}_{2} \mathrm{O}
\end{aligned}
$$

Die Messkurven zeigen, dass in allen Elektrolytlösungen sechs Hauptpeaks mit unterschiedlicher Intensität und Peaklage auftreten. Die Peaks bei $E=$ $+1,0 \mathrm{~V} \rightarrow+1,3 \mathrm{~V}$ in anodischer Richtung scheinen der Sauerstoffentwicklung sowie bei $\mathrm{E}=+0,9 \mathrm{~V} \rightarrow$ $+0,5 \mathrm{~V}$ der Reduktion der Oxidschicht zu entsprechen. Die Peaks für die Reduktion der Oxidschicht verschieben sich zu negativeren Werten, je geringer der $\mathrm{pH}-$ Wert ist. Der dem Peak bei $\mathrm{E}=+1,0 \mathrm{~V} \rightarrow$ $+1,3 \mathrm{~V}$ vorausgehende Basisstrom ist positiv und stellt neben der $\mathrm{O}_{2}$-Entwicklung die Bildung der PtOSchicht dar. Die Position des Oxidationspotentials von $\mathrm{Pt}$ relativ zum Peak bei $\mathrm{E}=+1,0 \mathrm{~V} \rightarrow+1,3 \mathrm{~V}$ stimmt mit dem von Cabelka et al. vorgeschlagenen elektrokatalytischen Mechanismus für die Oxidation von As(III) überein [12].

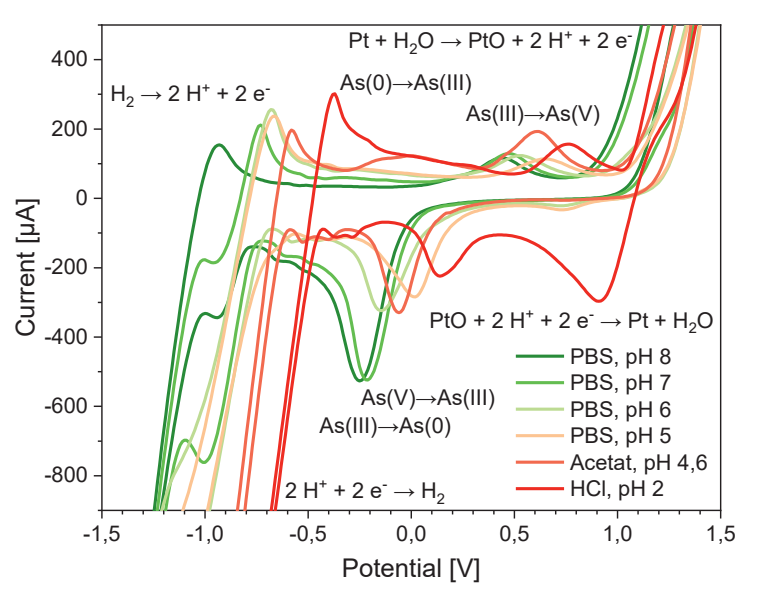

Abb. 2: Cyclovoltammetrische Untersuchung mit der angegebenen Elektrodenkonfiguration von As(III) für $\mathrm{c}(\mathrm{As}(\mathrm{III}))=1 \mathrm{mg} / \mathrm{l}$ in verschiedenen Pufferlösungen; Darstellung des jeweils 50 . CV-Zyklus.

Die Peaks bei $E=+0,3 \mathrm{~V} \rightarrow+0,8 \mathrm{~V}$ erscheinen bei einem hohen anodischen Potential, das der Oxidation von $\mathrm{As}(\mathrm{III}) \rightarrow \mathrm{As}(\mathrm{V})$ zugeordnet werden kann. Auf der kathodischen Seite des Voltammogramms verschwindet der hohe negative Strom bei $\mathrm{E}=-0,6 \mathrm{~V} \rightarrow$ -1,3 V, der die Wasserstoffentwicklung darstellt, oder er wird stark reduziert und zudem bei höherem $\mathrm{pH}-$ Wert zu negativeren Potentialen verschoben. Die Peaks bei $E=+0,2 \mathrm{~V} \rightarrow-0,2 \mathrm{~V}$ in kathodischer Richtung sowie bei $E=-0,3 \vee \rightarrow+0,2 \vee$ entsprechen der Reduktion von $\mathrm{As}(\mathrm{V}) \rightarrow \mathrm{As}(\mathrm{III})$ und $\mathrm{As}(\mathrm{III}) \rightarrow \mathrm{As}(0)$ bzw. der Oxidation von As(0) $\rightarrow$ As(III). Die Messergebnisse stimmen mit den Ergebnissen früherer Untersuchungen überein $[12,13]$. Außerdem verschiebt sich der kathodische Peak bei $\mathrm{E}=+0,2 \mathrm{~V} \rightarrow-0,2 \mathrm{~V}$ (Reduktion von PtO) mit Erniedrigung des pH-Werts zu höheren Potentialen. Dieser liegt nach der Oxidation von $\mathrm{As}(\mathrm{III}) \mathrm{zu} A \mathrm{~s}(\mathrm{~V})$ bei einem um etwa 0,3 V negativeren Potential vor. Das könnte durch eine langsame Reduktion desselben Zwischenprodukts, $\operatorname{PtOAs}(\mathrm{OH})_{3}$, erklärt werden, die im elektrokatalytischen Mechanismus vorgeschlagen wird. Es zeigte sich bei den verschiedenen verwendeten $\mathrm{pH}$ Werten, dass die Signalintensität bei der Oxidation von $\mathrm{As}(0) \rightarrow \mathrm{As}(\mathrm{III})$ unterschiedlich ist. Dieser scheint bei einem Acetatpuffer $(\mathrm{pH}=4,6)$ und Salzsäure $(\mathrm{pH}=2)$ intensiver zu sein, als bei den verwendeten PBS-Puffern. Dies könnte auf das Vorhandensein von mehr Arsen an der Platinelektrode bei niedrigerem $\mathrm{pH}-$ Wert zurückzuführen sein, das aus der Reduktion zu As(-III) bei dem niedrigeren Potential $(E=-0,6 \vee \rightarrow-1,3 \vee$ ) und der chemischen Anreicherung von mehr Arsen resultiert: As(-III) + $\mathrm{As}(\mathrm{III}) \rightarrow 2 \mathrm{As}(0)$ [14]. 


\section{Nachweis von As(III) durch ASV}

Nachfolgend werden SWASV-Untersuchungen zum direkten Nachweis von Arsenspezies sowie zur Signalverbesserung vorgenommen. Abb. 3 (a) zeigt die Auswirkung der Variation der As(III)Konzentration von $0-80 \mu \mathrm{g} / \mathrm{l}$ Arsen(III) bei $\mathrm{pH}=2,0$ in $0,01 \mathrm{~mol} / / \mathrm{HCl}$ bei einem Peak-Potential von $\mathrm{E}=$ $+0,06 \mathrm{~V}$. Bis zum Erreichen von $\mathrm{c}(\mathrm{As}(\mathrm{III}))=80 \mu \mathrm{g} / \mathrm{l}$ konnte eine hinreichende Linearisierung mit einer Signalintensität von $1,35 \mu \mathrm{A}$ erzielt werden. Infolgedessen zeigte sich bei gleichbleibender $\mathrm{c}(\mathrm{As}(\mathrm{III}))$ eine lineare Erhöhung des Peakstroms mit der Erhöhung von $\mathrm{c}(\mathrm{Cu}(\mathrm{II}))(\mathrm{Abb} .3(\mathrm{~b}))$. Für $\mathrm{c}(\mathrm{Cu}(\mathrm{II}))=100$ $\mu \mathrm{g} / \mathrm{l}$ wurde eine Signalintensität von $1,75 \mu \mathrm{A}$ und für $\mathrm{c}(\mathrm{Cu}(\mathrm{II}))=500 \mu \mathrm{g} / \mathrm{l}$ von $3,2 \mu \mathrm{A}$ erhalten. Dieser Trend zeigt zudem die Probleme, welche bei der Detektion von Arsenspezies auftreten können, wenn sie in Gegenwart von $\mathrm{Cu}$ (II) vorliegen. Andererseits können bei gleichbleibender Arsenkonzentration $\mathrm{Cu}$ (II) zur Intensitätserhöhung bspw. als Zusatz in Elektrolytlösungen genutzt werden. Neben dem charakteristischen Peak für $\mathrm{As}$ (III) konnte aber $\mathrm{Cu}$ (II) bei einem Peak-Potential von $E=-0,13 \vee$ gut getrennt von As(III) nachgewiesen werden.

(a)

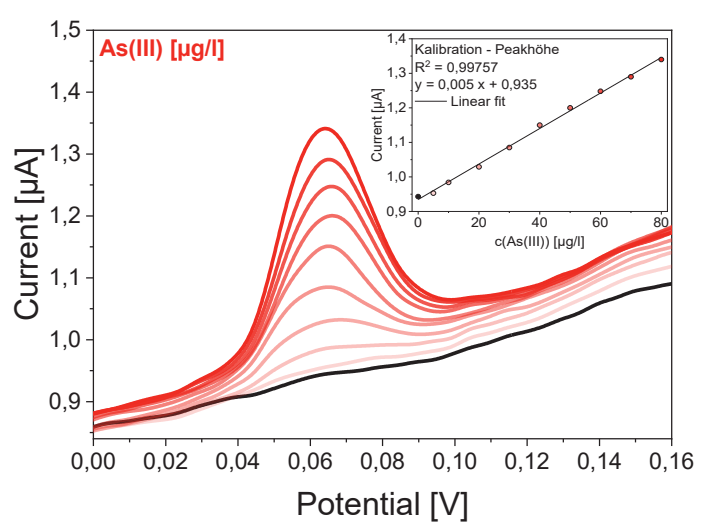

(b)

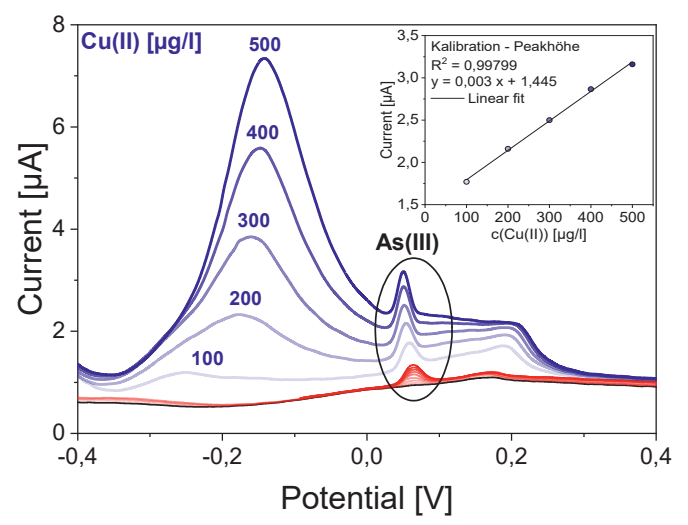

Abb. 3: Anodische-Stripping-Voltammogramme (a) für $0-80 \mu \mathrm{g} / \mathrm{I} \mathrm{As}(\mathrm{III})$ ohne $\mathrm{Cu}(\mathrm{II})$ und (b) für $80 \mu \mathrm{g} / \mathrm{I} \mathrm{As}(\mathrm{III})$ und Variation von $\mathrm{c}(\mathrm{Cu}(\mathrm{II}))=0-500 \mu \mathrm{g} / \mathrm{l}$ in $0,01 \mathrm{~mol} / \mathrm{l}$ $\mathrm{HCl}, \mathrm{pH}=2,0$. Der Ausschnitt zeigt die lineare Kalibrierungskurve die aus den entsprechenden Stripping-
Voltammogrammen extrahiert wurde; schwarze Linie: reine Elektrolytlösung.

\section{Nachweis von As(V) durch CSV}

Eine weitere Methode der Bestimmung von Arsenspezies ist die CSV. Das ist eine übliche Methode zur Bestimmung anorganischer und organischer Anionen. Bei diesen gibt es keine Anreicherungspotential bzw. -zeit und das Voltammogramm wird in kathodische Richtung aufgenommen. Für die "CoElektrolyse" wurde auch hier $\mathrm{Cu}$ (II) verwendet. Nach der Adsorption aus der $\mathrm{Cu}$ (II)-haltigen Lösung kann durch Oxidation oder Reduktion aus der intermetallischen Verbindung die Arsenspezies herausgelöst werden. Kathodische Voltammogramme zeigen in der Regel nur einen Peak, der im Einzelnen dem Übergang zum As(-III) entspricht. Zum Vergleich zeigen sich bei der anodischen Stripping-Analyse mehrere Peaks, die der Oxidation des Analyten, des Kupfers sowie weiterer Bestandteile in der Elektrolytlösung zuzuordnen sind. Der kathodische Verlauf von As(III) in einem Konzentrationsbereich von 0 $140 \mu \mathrm{g} / \mathrm{l}$ ist in Abb. 4 (a) dargestellt. Durch die Konzentrationserhöhung sind keine Potentialänderungen in den Voltammogrammen nachweisbar. Durch den Zusatz von $\mathrm{Cu}(\mathrm{II})$ in der Elektrolytlösung konnte nach nachfolgender Erhöhung von $\mathrm{c}(\mathrm{As}(\mathrm{III}))$ eine Signaländerung detektiert werden.

(a)

(b)
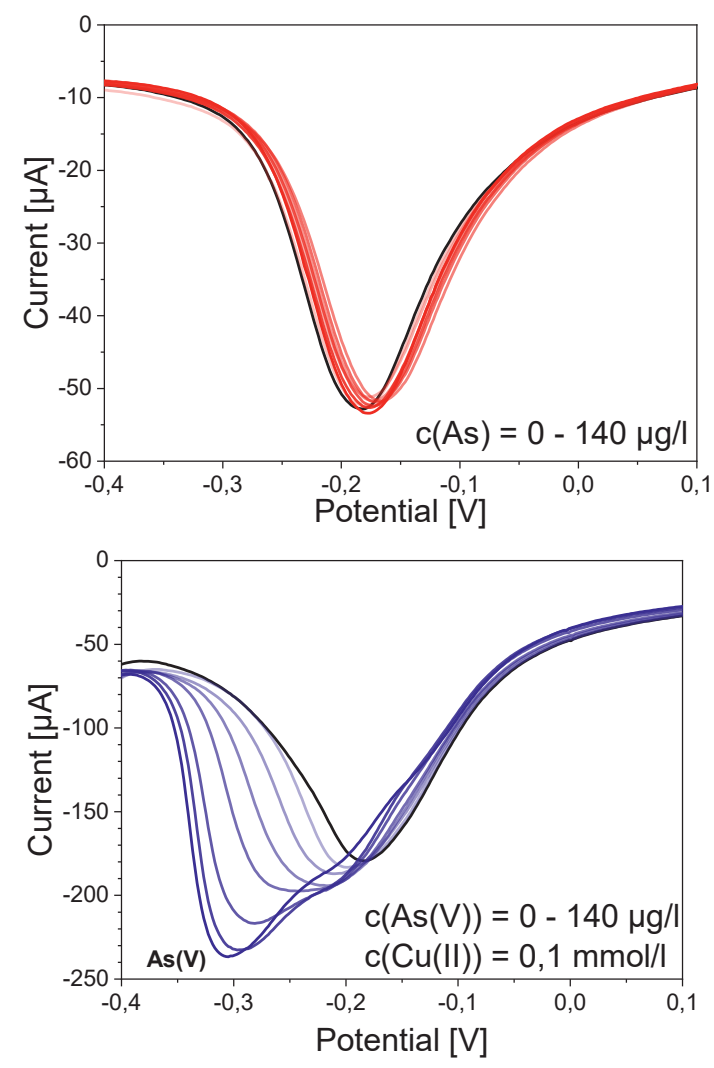

Abb. 4: Kathodische-Stripping-Voltammogramme (a) für $0-80 \mu \mathrm{g} / \mathrm{l} \mathrm{As}(\mathrm{III})$ ohne $\mathrm{Cu}(\mathrm{II})$ und (b) bei Zusatz von Kup- 
ferionen mit einer Konzentration von $\mathrm{c}(\mathrm{Cu}(\mathrm{II}))=0,1 \mathrm{mmol} / \mathrm{l}$ und Variation von $\mathrm{c}(\mathrm{As}(\mathrm{V})=20-140 \mu \mathrm{g} / \mathrm{l}$ in $\mathrm{PBS}, \mathrm{pH}=$ 7,0; schwarze Linie: reine Elektrolytlösung.

\section{Einfluss von Reduktionsmitteln auf die vorliegende Arsenspezies}

Der Einfluss von Ascorbinsäure auf die voltammetrischen Messungen sollte klären, inwieweit die vorliegende Arsenspezies durch ein zugesetztes Reduktionsmittel beeinflusst werden kann. Hierfür wurde eine Stammlösung aus $100 \mu \mathrm{g} / \mathrm{l}$ As(III) für mindestens 3 Monate gelagert um diese zur Oxidation zu bringen (s. Abb. 5 (a)). Nach der angegebenen Zeit ist eine deutliche Oxidation anhand der Voltammogramme in Form eines zunehmenden As(V)-Peaks bei $E=+0,48 \mathrm{~V}$ neben dem As(III)Peak bei $E= \pm 0 \mathrm{~V}$ sichtbar. Nach dem Zusatz von $10 \mathrm{mg} / \mathrm{l}$ Ascorbinsäure zu der Stammlösung und erneuter SWASV-Messung konnte kein As(V)-Peak bei $E=+0,48 \mathrm{~V}$ nachgewiesen werden. Stattdessen zeigte sich ein intensiverer, gut ausgeprägter linearer Verlauf beim As(III)-Peak (s. Abb. 5 (b)).

(a)

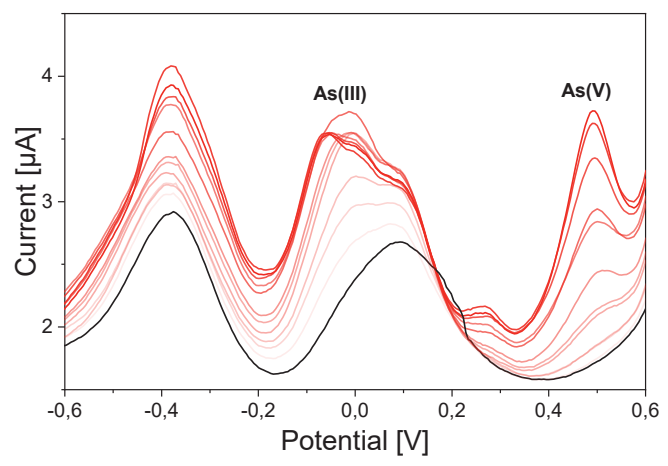

(b)

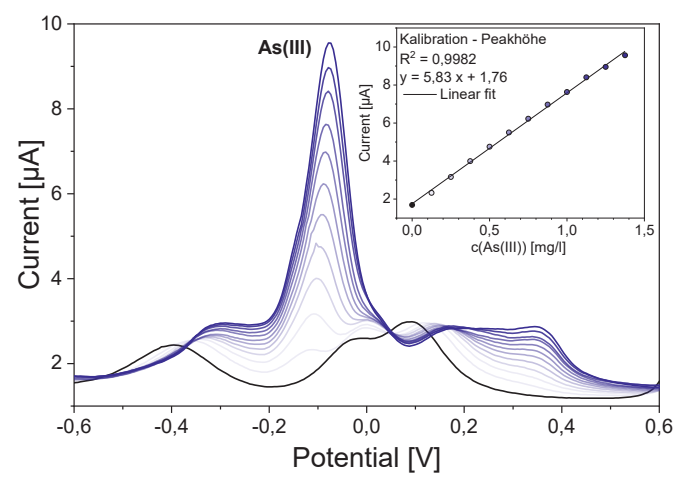

Abb. 5: Anodische-Stripping-Voltammogramme mit $c($ As $($ III $)=0,125-1,375 \mathrm{mg} / \mathrm{l}$ in Acetatpuffer, $\mathrm{pH}=4,6$; $E_{\text {dep }}=-1,0 \mathrm{~V} ; t_{\text {dep }}=300 \mathrm{~s}$ für $(\mathrm{a})$ ohne den Zusatz von Ascorbinsäure und (b) mit dem Zusatz von Ascorbinsäure $c(A A)=10 \mathrm{mg} / \mathrm{l}$. Der Ausschnitt zeigt die lineare Kalibrierungskurve die aus den entsprechenden StrippingVoltammogrammen extrahiert wurde; schwarze Linie: reine Elektrolytlösung.

\section{Diskussion}

Es wurde eine elektrochemische Untersuchung von Arsenspezies in sauren Lösungen mittels cyclischer Voltammetrie an einer Pt-Arbeitselektrode durchgeführt. Ein gut definierter Peak bei $E=+0,3 \rightarrow+0,8 \mathrm{~V}$ wird auf die Oxidation von $\mathrm{As}(\mathrm{III}) \rightarrow \mathrm{As}(\mathrm{V})$ zurückgeführt. Die entsprechende Reduktion von $\mathrm{As}(\mathrm{V})$ wird im Potentialbereich -0,6 - +1,2 V durch die Überlagerung von $\mathrm{As}(\mathrm{III}) \rightarrow \mathrm{As}(0)$ nicht beobachtet. $\mathrm{As}(\mathrm{V})$ ist in diesem Bereich vermutlich nicht elektrochemisch aktiv und hat keine Auswirkungen auf die Wasserstoffentwicklung. Mit höherem pH-Wert des Elektrolyten verschiebt sich die Wasserstoffentwicklung auf $\mathrm{Pt}$ in Richtung negativerer Potentiale und findet gleichzeitig mit der Arsinproduktion statt. Mit Hilfe der SWASV wurde das Verhalten von Arsenspezies durch die Zugabe von Cu(II) durch SWASV und CSV in wässrigen Lösungen untersucht. Die Untersuchungen decken einen Potentialbereich zwischen -1,5 und +1,5 V ab, wobei dem As(III)/As(V)Redoxprozess besondere Aufmerksamkeit gewidmet wurde. Es konnte gezeigt werden, dass durch den Zusatz von $\mathrm{Cu}(\mathrm{II})$ die Signalintensität von Arsenspezies in Lösung erhöht werden konnte. Bei der SWASV konnten gut getrennte Peaks für As(III) (E = $+0,06 \mathrm{~V})$ und $\mathrm{Cu}(\mathrm{II})(\mathrm{E}=-0,13 \mathrm{~V}$ ) nachgewiesen werden und ermöglichten so eine genaue und reproduzierbare Kodierung. Hierdurch zeigt sich die Möglichkeit noch geringere Nachweisgrenzen $(\leq 20$ $\mu \mathrm{g} / \mathrm{I} \mathrm{As}(\mathrm{III}))$ für die Bestimmung von As zu erreichen. Bei der CSV konnte dagegen nur ein Peak nachgewiesen werden. Durch die Verwendung eines Reduktionsmittels konnte signifikant das Vorliegen der Arsenspezies hin zu As(III) beeinflusst werden. Diese Ergebnisse zeigen, dass die ASV oder die CSV für einen sicheren, schnellen Vor-Ort-Nachweis von $\mathrm{As}(\mathrm{III})$ und $\mathrm{Cu}(\mathrm{II})$ in realen Wasserproben geeignet sind.

\section{Literatur}

[1] HÖSEL, G.; HOTH, K.; JUNG, D.; LEONHARDT, G.; MANN, M.; MEYER, H.; TÄGL, U. (1994): Das Zinnerz-Lagerstättengebiet Ehrenfriedersdorf/Erzgebirge, Bergbaumonographie, Bergbau in Sachsen Band 1; Hrsg: LFUG und Oberbergamt, Freiberg.

[2] PETRUSEVSKI, B., SHARMA, S., SCHIPPERS, J. C., \& SHORDT, K. (2007). Arsenic in drinking water. Delft: IRC International Water and Sanitation Centre, 17(1), 36-44.

[3] NAIDU, R., SMITH, E., OWENS, G., \& BHATTACHARYA, P. (2006). Managing arsenic in the environment: from soil to human health. CSIRO publishing.

[4] RAVENSCROFT, P., BRAMMER, H., \& RICHARDS, K. (2009). Arsenic Pollution: A Global Synthesis, RGS- 
IBG Book Series, A John Wiley and Sons Ltd. Publication, London.

[5] NRIAGU, J. O., BHATTACHARYA, P., MUKHERJEE, A. B., BUNDSCHUH, J., ZEVENHOVEN, R., \& LOEPPERT, R. H. (2007). Arsenic in soil and groundwater: an overview. Trace Metals and other Contaminants in the Environment, 9, 3-60.

[6] BEDNAR, A. J., GARBARINO, J. R., RANVILLE, J. F., \& WILDEMAN, T. R. (2005). Effects of iron on arsenic speciation and redox chemistry in acid mine water. Journal of Geochemical Exploration, 85(2), 55-62.

[7] SAMADZADEH YAZDI, M. R., \& KHODADADI DARBAN, A. (2010). Effect of arsenic speciation on remediation of arsenic-contaminated soils and waters.

[8] SCHWARZ, J., TROMMER, K., \& MERTIG, M. (2016). Novel Screen-Printed All-Solid-State Copper (II)Selective Electrode for Mobile Environmental Analysis. American Journal of Analytical Chemistry, 7(07), 525.

[9] SCHWARZ, J., TROMMER, K., \& MERTIG, M. (2018). Solid-contact ion-selective electrodes based on graphite paste for potentiometric nitrate and ammonium determinations. American Journal of Analytical Chemistry, 9(12), 591.

[10] SCHOLZ, S.: Qualifizierung eines VoltammetrieMesssystems, Freie Universität Berlin, Dissertation, 2013.

[11] BRUSCIOTTI, F., \& DUBY, P. (2007). Cyclic voltammetry study of arsenic in acidic solutions. Electrochimica acta, 52(24), 6644-6649.

[12] CABELKA, T. D., AUSTIN, D. S., \& JOHNSON, D. C. (1984). Electrocatalytic Oxidation of As (III): I. Voltammetric Studies at Pt Electrodes in. Journal of the Electrochemical Society, 131(7), 1595.

[13] WEI, Z., \& SOMASUNDARAN, P. (2004). Cyclic voltammetric study of arsenic reduction and oxidation in hydrochloric acid using a Pt RDE. Journal of applied electrochemistry, 34(2), 241-244.

[14] REEVES, R. M. (1978). , AJ Bard (Ed.) Encyclopedia of Electrochemistry of the Elements, Vol. VII, Dekker, New York, NY (1976).

\section{Danksagung}

Das dieser Arbeit zugrunde liegende Vorhaben wird mit Mitteln des Bundesministeriums für Bildung und Forschung im Programm „WIR! - Wandel durch Innovation in der Region" unter dem Förderkennzeichen 03WIR1906E gefördert und im Rahmen des Projektes „rECOmine - Ressourcenorientierte Umwelttechnologien für das 21. Jahrhundert" umgesetzt. Das Kurt-Schwabe-Institut für Mess- und Sensortechnik Meinsberg e.V. wird mitfinanziert durch Steuermittel auf der Grundlage des vom Sächsischen Landtag beschlossenen Haushaltes. 